ESJ Social Sciences

\title{
Activités coopératives et acquisition de compétences sociales par des adolescents auteurs de viol du centre d'observation des Mineurs de Bouake (RCI)
}

\author{
Sadia Martin Armand, Docteur
}

Université Alassane Ouattara/Bouake, Côte d'Ivoire

Doi:10.19044/esj.2021.v17n27p307

Submitted: 26 June 2021

Accepted: 27 July 2021

Published: 31 August 2021
Copyright 2021 Author(s)

Under Creative Commons BY-NC-ND

4.0 OPEN ACCESS

Cite As:

Sadia M.A. (2021). Activités coopératives et acquisition de compétences sociales par des adolescents auteurs de viol du centre d'observation des Mineurs de Bouake (RCI). European Scientific Journal, ESJ, 17 (27), 307. https://doi.org/10.19044/esj.2021.v17n27p307

\section{Résumé}

En quoi les activités coopératives influent-elles sur l'acquisition de compétences sociales par les adolescents auteurs de viol ? Telle est la question qui a suscité cette étude réalisée au Centre d'Observation des Mineurs (COM) de Bouaké. L'objectif visé est d'évaluer les influences des activités coopératives sur l'acquisition des compétences sociales par les adolescents privés de liberté. L'hypothèse principale postule que «plus les enfants délinquants auteurs de viol ont des activités coopératives, plus ils acquièrent des compétences sociales ». L'ancrage théorique de cette étude est un modèle interactionniste qui s'inspire de l'interactionnisme sociale de Vygotsky et Brunner et de la théorie de l'interdépendance sociale de Deutsch $(1949,1962)$. Pour collecter les données, l'on a eu recourt à la méthode comparative et aux techniques de l'observation structurée autour de trois grilles et de la recherche documentaire. Les principaux résultats obtenus révèlent que les activités coopératives influent sur les comportements sociaux, l'adoption de comportements responsables et enfin, la capacité de travailler en équipe des sujets d'un temps $\mathrm{T} 1$ à un temps $\mathrm{T} 2$. Ces résultats confirment ainsi l'hypothèse générale formulée en ce sens qu'ils ont établi que la coopération est un outil de «facilitation sociale pour désigner l'effet positif du groupe, sur les acquisitions des personnes le constituant ». 
Mots clés : Coopération - compétences sociales - adolescents - viol relations sociales - équipe - Côte d'Ivoire

\title{
Cooperative Activities and Social Skills Training for Adolescent Rapists at the Bouake Juvenile Observation Center (RCI)
}

Sadia Martin Armand, Docteur

Université Alassane Ouattara/Bouake, Côte d'Ivoire

\begin{abstract}
How do cooperative activities affect the acquisition of social skills by adolescent rapists? This is the question that prompted this study conducted at the Centre d'Observation des Mineurs (COM) in Bouaké. The objective is to evaluate the influence of cooperative activities on the acquisition of social skills by adolescents deprived of their liberty. The main hypothesis postulates that "the more delinquent children engage in cooperative activities, the more social skills they acquire". The theoretical anchor of this study is an interactionist model that draws on Vygotsky and Brunner's social interactionism and the theory of social interdependence of Deutsch's (1949, 1962). To collect the data, we used the comparative method and the techniques of observation structured around three grids and documentary research. The main results obtained reveal that cooperative activities influence social behaviors, the adoption of responsible behaviors and finally, the ability to work in teams of the subjects from time T1 to time T2. These results confirm the general hypothesis formulated in the sense that they established that cooperation is a tool of "social facilitation to designate the positive effect of the group, on the acquisitions of the people constituting it".
\end{abstract}

Keywords: Cooperation - social skills - adolescents - rape - social relations team - Ivory Coast

\section{Introduction}

La mise en œuvre la primauté de l'éducatif sur la répression, affirmée par le titre X de la loi loi $\mathrm{n}^{\circ}$ 60-366 du 14 novembre 1960 portant code de procédure pénale et les articles 116 de la loi $\mathrm{n}^{\circ} 81-640$ du 31 juillet 1981portant code pénal, a conduit selon les articles 770 et suivant permettent le placement sous ordonnance de garde provisoire dans les Centres d'Observation des Mineurs (COM).

l'arrêté $n^{\circ} 642 / M J D H L P / C A B$ du 29 décembre 2015 portant organisation et fonctionnement de la Direction de la Protection judiciaire de l'Enfance et de la 
Jeunesse qui consacre son existence juridique. Selon l'article 19 de ce texte, "les COM ont pour mission d'accueillir, d'observer le comportement des mineurs, d'évaluer leurs potentialités et de rendre un avis d'orientation au juge des enfants afin de l'aider à prendre une décision dans l'intérêt supérieur du jeune. La prise en charge des mineurs placés au COM doit permettre de prévenir la récidive ». Pour y parvenir, les COM « organisent les loisirs des mineurs, recherchent les adresses des parents ou des répondants ». Il s'agit d'une prise en charge institutionnelle assujettie à une action éducative. Le but étant de favoriser la rééducation et la réinsertion sociale, familiale et professionnelle des mineurs en conflit avec la loi sur la base d'une observation approfondie ( Sadia, 2019).

Au COM de Bouaké, les enfants qui y séjournent ont commis pour la plupart des infractions criminelles, dont le viol. Quand, ils sont placés en détention préventive, ceux-ci ont des rapports difficiles avec leurs copensionnaires et même les éducateurs. En Août 2018, trois mineurs placés pour assassinat, se sont organisés pour tuer l'un de leur encadreur qu'ils jugeaient peu compréhensif. En 2019, précisément au mois de février, une bande de mineurs auteurs de viol a enclenché une grève de faim avec une tentative d'évasion massive. Ces comportements atypiques des enfants auteurs d'actes criminels a conduit l'équipe éducative à envisager un dispositif hétérogène destiné à les soustraire de leur isolement ou repli identitaire. Ainsi, 6 de ces adolescents auteurs de viol ont été associés à d'autres enfants pour exécuter les tâches afférentes à l'élevage de lapin. Le but de cette orientation dans une activité qui nécessite la coopération, est de réduire leur agressivité et par là même, facilité l'installation des compétences sociales. Mais qu'entendt-on par apprentissage coopératif (AC) et compétences sociales ?

L'apprentissage coopératif, il vise à «créer des conditions pour que des apprenants apprennent ensemble, entre eux, sous l'impulsion d'un formateur » (Dennery, 2014). A travers cette définition opérationnelle, c'est tout le caractère social de ce type d'apprentissage qui est ainsi mis en évidence. Les premiers chercheurs qui sont à la base de ce concept sont des américains selon Panitz (1999). Il s'agit de Lewin (1944) et de Dewey (1927), dont les travaux ont respectivement porté sur la dynamique de groupes, et sur la nature sociale des apprentissages qui suggère « la réflexion collective » (Baudrit, 2007).

Plutard dans les années 80, des chercheurs en psychologie sociale génétique vont développer ce concept. Il met en évidence l'influence positive des interactions sociales sur l'apprentissage. L'apprentissage entre pairs peut être supérieur, sous certaines conditions, à l'apprentissage seul ou face à un formateur car il suscite des confrontations de point de vue générant la remise en cause de représentations, et par conséquent l'émergence de connaissances nouvelles. Il invite les formateurs à favoriser les apprentissages coopératifs. (Dennery). 
L'apprentissage coopératif ainsi présenté exige le respect de quatre critères essentiels, rappelés par Dyson et Casey (2012). Ces groupes doivent avoir :

- un but commun,

- une interdépendance des actions,

- des interactions en face à face,

- des membres du groupe incités à s'entraider et s'encourager.

Quant aux compétences sociales, elles relèvent « de la socialisation et qui visent le développement de l'autonomie des élèves ainsi que le vivre ensemble » (F. Agathe, 2017, p. 169). Selon cet auteur, « elles se traduisent en comportements efficaces et appropriés, et se réfèrent aux normes et valeurs du ou des cadres de socialisation de l'individu. Les compétences sociales relèvent à la fois de la gestion de soi, de ses émotions et de la gestion des relations à autrui ». Ainsi, pour Sophie (2015), les compétences sociales sont « comportementales » et se déclinent en deux modalités. Il s'agit des :

" compétences sociales intra individuelles (comportements vis-à-vis de soi) et les compétences sociales interindividuelles (comportements vis-à-vis des autres). Ainsi, seront pris en compte à la fois des savoir-être internes à l'élève (compétences sociales intra-individuelles : comportements vis-à-vis de soi : ténacité, curiosité, autonomie, motivation, estime de soi ...) et des savoir être externes (compétences sociales interindividuelles : comportements vis-à-vis des autres : respect des règles de communication, de politesse, respect des règles de vie en communauté) ou se révélant dans l'interaction avec autrui ». Ces compétences ainsi définies ont une incidence "sur la réussite des individus sur le marché du travail, en termes d'insertion, d'employabilité ou encore de revenus (...) et sur la réussite des élèves » (Agathe, 2016). adolescents?

Comment parvenir à développer les compétences sociales chez les

En réponse à cette interrogation, Sylvain (2016) « met surtout en avant le potentiel des pratiques du plan de travail à développer l'autonomie, les comportements responsables et la coopération entre élèves». Pour cet auteur, «l'objectif n'est pas d'être meilleur que l'autre, ce qui n'a aucun intérêt, mais meilleur que soi».

Quel est l'état des lieux de la littérature scientifique sur l'apprentissage coopératif et l'acquisition des compétences sociales dans les structures privatives de liberté en Côte d'Ivoire ?

La littérature scientifique sur la prise en charge éducative de l'enfant incarcéré reste suffisamment muette sur l'application de la pédagogie coopérative. Les études en lien avec l'enfance délinquantes portent soit sur les 
conditions de détentions (Koudou, 2005), soit sur leurs incidences sur le développement et l'échec de la réinsertion de l'enfant (Koudou, 1994, Sadia, 2014). D'autres études par contre, portent sur les facteurs explicatifs de la conduite déviante et délinquante des mineurs en lien avec soit la famille d'origine ( Koudou, 2008) ou le milieu institutionnelle (Sadia, 2014). Enfin, les incidences de la stigmatisation verbale (Koudou, 1997 ; Koudou.1993). Malgré leur apport dans la compréhension du comportement délinquant des adolescents incarcérés, ces travaux semblent occulter l'incidence de la coopération sur le processus de rééducation des adolescents dits en conflit avec la loi. D'où la question suivante : en quoi les activités coopératives influentelles sur l'acquisition de compétence sociales par les adolescents auteurs de viol ? L'objectif de cette étude est d'évaluer les influences des activités coopératives sur l'acquisition des compétences sociales par les adolescents privés de liberté. Le postulat principal de cette étude est le suivant : plus les enfants délinquants ont des activités coopératives, plus ils acquièrent des compétences sociales. De cette hypothèse générale émergent trois hypothèses spécifiques :

- L'implication des mineurs dans des activités coopératives influe sur la qualité des relations sociales du groupe;

- L'acceptation des contradiction au cours des activités coopératives contribue à l'adoption de comportements responsables par les mineurs;

- Plus les enfants interagissent dans la réalisation des tâches, plus ils développent la capacité à travailler en équipe.

L'ancrage théorique de cette étude est un modèle interactionniste qui s'inspire de l'interactionnisme sociale de Vygotski et Brunner et de la dimension coopérative de la théorie de l'interdépendance sociale de Deutsch (1949, 1962). Pour les premiers auteurs cités, c'est dans les interactions sociales que se font les acquisitions cognitives et sociales. La deuxième quant à elle, met l'accent sur les effets différentiels de la coopération (..) sur les relations entre les personnes. Pour lui, la coopération intensifie les échanges et incite à l'entraide. Elle favorise « une résolution constructive des désaccords entre deux ou plusieurs personnes » (Deutsch, 1969, 1973). Quelle est la méthodologie choisie pour conduire cette étude?

\section{Méthodologie \\ 1. Site et population}

Le terrain de cette étude est le Centre d'Observation des Mineurs (COM) de Bouaké. Cette structure placée sous la tutelle de la Direction de la Protection judiciaire de l'enfance et de la Jeunesse. Elle est logée dans un bâtiment situé précisément, entre le Tribunal de Première Instance de Bouaké, non loin du Palais du carnaval. 
Sa population est constituée d'individus de sexe masculin, dont l'âge se situe entre 14 ans et 18 ans. Ils sont tous auteurs de diverses infractions à la loi pénale ivoirienne. L'effectif théorique de ce centre est de 25 enfants. Toutefois, dans la pratique, l'on dénombre en février 2021 au total 41pensionnaires. Les cinq adolescents observés ont été choisis sur la base du critères liés à l'infraction commise qui doit être un acte criminel (viol, assassinat, homicide etc.) et aux comportements asociaux après leur placement en détention. L'échantillonnage est non probabiliste et précisément, il s'agit de la technique du choix raisonné.

\section{Méthodes et techniques de collecte de données}

Nous avons opté pour la méthode comparative. Les comportements des enfants ont été observés sur deux périodes. La première part d'octobre 2019 à janvier 2020, et se situe dans la période avant l'ouverture de l'activité d'élevage du lapin. Puis, la seconde période, a débuté de fin janvier pour s'estomper en fin Mai 2020, correspond au moment où ces enfants ont été placés en situation de coopération avec les autres. Sur chacune de ces périodes, les comportements des adolescents privés de liberté ont été observés et consignés dans des grilles. Ainsi, deux techniques ont permis la collecte de données : l'observation et la recherche documentaire, notamment les rapports d'observation et d'incidents.

Les trois grilles ont été conçus autour de trois sous-thèmes et d'items inspirés des catégories de Balse, notamment les réactions positives (Abric, 2019) et de la synthèse des compétences sociales scolaires de N. Epinoux et Lafont, (2014). La première évalue la qualité des relations sociales entre les mineurs ciblés. La deuxième, quant à elle, est relative au comportement responsable. Enfin, la dernière grille porte sur la capacité à travailler en équipe. Chacune d'elles comporte 5 items annotés $(+)$ quand le sujet réalise la compétence requise et (-) quand il n'y parvient pas. Chaque réaction observée (positive ou négative) comptabilise 1point. A la fin, nous avons fait la somme de chaque catégorie d'observation. A partir de ces données, les moyennes et écart type ont été calculés. Sur la base de ces données descriptives, l'on a procédé à l'étude des inférences statistiques en mettant en corrélation les variables apprentissages coopératives et acquisition des compétences sociales. Comment ces différentes grilles ont-elles été annotées ?

Nous avons par nous-même utilisé ces grilles pour observer les adolescents. Ensuite, au cours de la séance de rédaction du rapport trimestrielle d'observation, nous avons confronté nos données à celles des éducateurs pour ne retenir que les points qui sont consensuels. 


\section{Méthode d'analyse de données}

Les données ont fait l'objet d'une analyse descriptive dans un premier temps. Puis, dans le second temps, et dans l'optique d'évaluer le lien entre la coopération et l'acquisition de compétences prosociales, il a été procédé au calcul du $\mathrm{T}$ de Student, un test d'hypothèse qui a contribué à comparer les moyennes de 2 séries appariées. Le T de student est calculé grâce au logiciel XLSTAT version 2018.5.

\section{Résultats}

3.1. Présentation et analyse des résultats

Les principaux résultats de cette étude portent donc sur les différentes synthèses des observations relatives à la qualité des relations avec les condisciples, aux comportements responsables et à la capacité à travailler en équipe. 
Tableau $\mathbf{n}^{\circ} 1$ : Synthèse des observations sur la qualité des relations sociales avec les condisciples

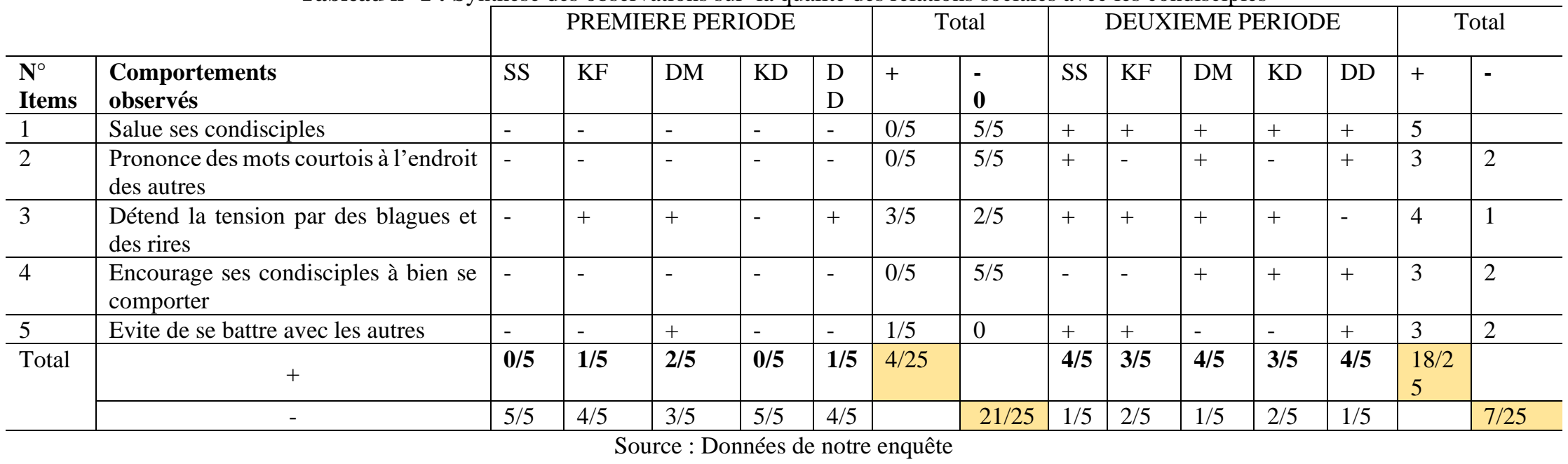

Selon la grille ci-dessus, à la première période de notre étude, aucun enfant n'est parvenu à développer les compétences attendues aux items 1, 2 et 4 . En ce qui concerne les sujets observés, l'on note que SS et KD n'ont réalisé aucune action prévue par la compétence qualité de la relation. Quant aux mineurs KF, DM et DD, ils parviennent à « détendre la tension par des blagues et des rires ». A la deuxième période, les mineurs parviennent à appliquer les comportements prévus aux items 1 et 3 . En outre, le score individuel de ces enfants oscille entre 3 et 4 sur 5 . Hormis, KF et $\mathrm{KD}$, les sujets SS, DM et DD appliquent 4 items sur les 5 évalués. 
Ces résultats descriptifs ne permettant pas de conclure statistiquement que la coopération influe sur les compétences sociales des enfants privés de liberté. C'est à cette préoccupation que répond le tableau suivant :

Tableau n $^{\circ}$ 2: lien entre coopération et qualité des relations avec les autres du T1 au T2

\begin{tabular}{c|r|r|r} 
Résultats & TEMPS T1 & TEMPS T2 & Différence \\
\hline Nb valides & 5 & 5 & 5 \\
\hline Moyenne & 0,8 & 3,6 & $-2,8$ \\
Ecart-Type & 0,837 & 0,548 & 0,289 \\
\hline Médiane & 1 & 4 & -3 \\
Quartile 25\% & 0 & 3 & -3 \\
Quartile 75\% & 1 & 4 & -2 \\
Inter Quartile & 1 & 1 & 1
\end{tabular}

Ces variations ainsi décrites, permettent-elles de conclure en l'existence de lien entre les deux variables étudiées?

Résultats de la comparaison des moyennes de 2 séries appariées :

Les effectifs sont insuffisants pour baser le test sur le calcul de l'écart-réduit, on utilisera la valeur de $\mathrm{t}$ student. $\mathrm{t}$ $(25)=0.62 ; \mathrm{p}=0,0017$

Conclusions du test de comparaison des moyennes de 2 séries appariées :

Les conditions d'applications du test sont respectées (distributions des écarts selon la loi normale). La valeur du t permet d'observer que les moyennes des 2 séries de mesures sur le même groupe sont significativement différentes. Autrement dit, la coopération influe sur la qualité des relations entre les adolescents d'un temps T1 à un temps T2. 
Tableau $^{\circ} 3$ : Synthèses des grilles d'observation sur les comportements responsables

\begin{tabular}{|c|c|c|c|c|c|c|c|c|c|c|c|c|c|c|c|}
\hline \multirow[b]{2}{*}{$\begin{array}{l}\mathbf{N}^{\circ} \\
\text { Items }\end{array}$} & \multirow[b]{2}{*}{$\begin{array}{l}\text { Comportements } \\
\text { observés }\end{array}$} & \multicolumn{5}{|c|}{ PREMIERE PERIODE } & \multicolumn{2}{|c|}{ Total } & \multicolumn{5}{|c|}{ DEUXIEME PERIODE } & \multicolumn{2}{|c|}{ Total } \\
\hline & & SS & KF & $\mathrm{DM}$ & KD & $\mathrm{DD}$ & + & - & SS & KF & $\mathrm{DM}$ & KD & DD & + & - \\
\hline 1 & Ne perturbe pas la sieste des autres; & - & - & - & - & - & $0 / 5$ & $5 / 5$ & + & - & + & - & - & $2 / 5$ & $3 / 5$ \\
\hline 2 & $\begin{array}{l}\text { Lave ses habits et maintien sa cellule } \\
\text { propre }\end{array}$ & - & - & - & - & - & $0 / 5$ & $5 / 5$ & + & + & + & + & - & $4 / 5$ & $1 / 5$ \\
\hline 3 & $\begin{array}{l}\text { Sert à manger aux autres sans aucune } \\
\text { discrimination }\end{array}$ & - & + & + & - & + & $3 / 5$ & $2 / 5$ & + & - & + & + & + & $4 / 5$ & $1 / 5$ \\
\hline 4 & $\begin{array}{l}\text { Adhère à la position de la majorité pour } \\
\text { éviter des conflits }\end{array}$ & - & - & + & - & - & $1 / 5$ & $5 / 5$ & + & + & - & + & + & $4 / 5$ & $1 / 5$ \\
\hline 5 & $\begin{array}{l}\text { Demande pardon quand il commet une } \\
\text { faute }\end{array}$ & - & - & - & - & - & $0 / 5$ & $4 / 5$ & + & + & + & + & + & $5 / 5$ & $0 / 5$ \\
\hline \multirow[t]{2}{*}{ Total } & + & $0 / 5$ & $1 / 5$ & $2 / 5$ & $0 / 5$ & $1 / 5$ & $4 / 25$ & & $5 / 5$ & $4 / 5$ & $4 / 5$ & $4 / 5$ & $4 / 5$ & $19 / 25$ & \\
\hline & - & $5 / 5$ & $4 / 5$ & $3 / 5$ & $5 / 5$ & $4 / 5$ & & $21 / 25$ & $0 / 5$ & $0 / 5$ & $0 / 5$ & $0 / 5$ & $0 / 5$ & & $6 / 2$ \\
\hline
\end{tabular}

Source : Données de notre enquête

Il ressort de ce tableau que sur toute la durée de la première période, tous les enfants se sont abstenus de réaliser les comportements attendus aux items 1,2 et 5. Chez les enfants, 1'on note que SS et KD n'ont réalisé aucune action prévue par les propositions. L'item qui a reçu le plus d'adhésion des sujets est le point 3 . A la deuxième période, seul le mineur SS (5/5). Tous les autres ont connu un succès avec un score de 4/5. L'incidence statistique de cette progression, sera évaluée par le test du T de Student selon le tableau ci-après : 
Tableau $n^{\circ} 4$ : coopération et comportements responsables

\begin{tabular}{c|r|r|r} 
Résultats & TEMPS T1 & TEMPS T2 & Différence \\
\hline Nb valides & 5 & 5 & 5 \\
\hline Moyenne & 0,8 & 4,2 & $-3,4$ \\
Ecart-Type & 0,837 & 0,447 & 1,140 \\
\hline Médiane & 1 & 4 & -3 \\
Quartile 25\% & 0 & 4 & -4 \\
Quartile 75\% & 1 & 4 & -3 \\
Inter Quartile & 1 & 0 & 1
\end{tabular}

Source: Données de 1'enquête

Résultats de la comparaison des moyennes de 2 séries appariées :

Les effectifs sont insuffisants pour baser le test sur le calcul de l'écart-réduit, on utilisera la valeur de $\mathrm{t}$ student $\mathrm{t}$ $(25)=0.82$ et $\mathrm{p}=0,0026$

Les conditions d'applications du test sont respectées (distributions des écarts selon la loi normale)

La valeur du $\mathrm{t}$ permet d'affirmer que les moyennes des 2 séries de mesures sur le même groupe sont significativement différentes. En d'autres termes, les moyennes relatives aux comportements responsables sur les deux périodes de l'étude sont significativement différentes. 
Tableau $\mathbf{n}^{\circ} 5$ : Grille d'observation de la capacité à travailler en équipe

\begin{tabular}{|c|c|c|c|c|c|c|c|c|c|c|c|c|c|c|c|}
\hline & & \multicolumn{5}{|c|}{ PREMIERE PERIODE } & \multicolumn{2}{|c|}{ Total } & \multicolumn{5}{|c|}{ DEUXIEME PERIODE } & \multicolumn{2}{|c|}{ Total } \\
\hline $\begin{array}{l}\mathbf{N}^{\circ} \\
\text { Items }\end{array}$ & $\begin{array}{l}\text { Comportements } \\
\text { observés }\end{array}$ & SS & KF & $\mathbf{D M}$ & KD & DD & + & - & SS & KF & DM & KD & DD & + & - \\
\hline 1 & $\begin{array}{l}\text { S'implique dans la réalisation } \\
\text { des travaux d'intérêt général ; }\end{array}$ & - & - & - & - & - & $\mathrm{C}$ & $5 / 5$ & + & + & + & + & + & $5 / 5$ & $0 / 5$ \\
\hline 2 & $\begin{array}{l}\text { Fais preuve } \\
\text { d'initiative }\end{array}$ & - & - & - & - & - & $0 / 5$ & $5 / 5$ & + & - & + & - & + & $3 / 5$ & $2 / 5$ \\
\hline 3 & $\begin{array}{l}\text { Délaisse ses activités } \\
\text { personnelles au profit des } \\
\text { travaux de groupe }\end{array}$ & - & + & - & - & + & $2 / 5$ & $3 / 5$ & + & + & + & + & - & $3 / 5$ & $2 / 5$ \\
\hline 4 & $\begin{array}{l}\text { Laisse les autres exprimer leur } \\
\text { point de vue }\end{array}$ & - & - & - & - & - & $0 / 5$ & $5 / 5$ & - & - & + & + & + & $3 / 5$ & $2 / 5$ \\
\hline 5 & $\begin{array}{l}\text { Aide les autres à laver leurs } \\
\text { cellules }\end{array}$ & - & - & + & - & - & $1 / 5$ & $4 / 5$ & + & + & - & + & - & $3 / 5$ & $2 / 5$ \\
\hline Total & + & $0 / 5$ & $1 / 5$ & $1 / 5$ & $0 / 5$ & $1 / 5$ & $3 / 25$ & & $4 / 5$ & $3 / 5$ & $4 / 5$ & $4 / 5$ & $3 / 5$ & $18 / 25$ & \\
\hline & - & $5 / 5$ & $4 / 5$ & $4 / 5$ & $5 / 5$ & $4 / 5$ & & $22 / 25$ & $1 / 5$ & $2 / 5$ & $1 / 5$ & $1 / 5$ & $2 / 5$ & & $7 / 25$ \\
\hline
\end{tabular}

Ce tableau permet d'observer que sur toute la durée de la première période, les items 1,2 et 4 n'ont pas été réalisés par tous les enfants. Quant à l'item 5 seul, le mineur DM développe cette compétence dans ses relations aux autres à la première période. On observe également, que SS et KD n'ont réalisé aucune action prévue par les propositions. A la deuxième période, les mineurs SS, DM et KD totalise 4 points sur les 5 attendus. Quand à KF et DD, ils sont passés respectivement d'un score de $0 / 5$ et de $1 / 5$ à $3 / 5$ chacun. Mais, ces évolutions sont-elles statiquement significatives ? 
Pour évaluer les influences de la coopération sur la capacité de travailler en équipe des 5 sujets, le test de T de Student a été convoqué. Deux hypothèses sont formulées à cet effet :

Tableau $\mathbf{n}^{\circ} \mathbf{6}$ : Lien entre activités coopérative et capacité à travailler en équipe

\begin{tabular}{c|r|r|r} 
Résultats & TEMPS T1 & TEMPS T2 & Différence \\
\hline Nb valides & 5 & 5 & 5 \\
\hline Moyenne & 0,6 & 3,6 & -3 \\
Ecart-Type & 0,548 & 0,548 & 1 \\
\hline Médiane & 1 & 4 & -3 \\
Quartile 25\% & 0 & 3 & -4 \\
Quartile 75\% & 1 & 4 & -2 \\
Inter Quartile & 1 & 1 & 2
\end{tabular}

Source: Données de l'enquête

Résultats de la comparaison des moyennes de 2 séries appariées :

Les effectifs sont insuffisants pour baser le test sur le calcul de l'écartréduit, on utilisera la valeur de t student $\mathrm{t}(25)=0.86 ; \mathrm{p}=0,0026$.

Les conditions d'applications du test sont respectées (distributions des écarts selon la loi normale).

La valeur du t révèle que les moyennes des 2 séries de mesures sur le même groupe sont significativement différentes. En d'autres termes, la capacité de travailler en équipe change selon que les enfants sont impliqués dans une activité de rééducation basée sur la coopération.

\subsection{Discussion}

L'objectif de cette étude est d'évaluer les influences des activités coopératives sur l'acquisition des compétences sociales par les adolescents privés de liberté. L'hypothèse principale postule que "plus les enfants délinquants ont des activités coopératives, plus ils acquièrent des compétences sociales ». L'ancrage théorique de cette étude est un modèle interactionniste qui s'inspire de l'interactionnisme sociale de Vygotski et Brunner et de la dimension coopérative de la théorie de l'interdépendance sociale de $\mathrm{M}$. Deutsch $(1949,1962)$. Les principaux résultats obtenus révèlent premièrement que les activités coopératives influent sur la qualité des relations sociales avec les autres, l'adoption de comportements responsables et enfin, la capacité à travailler en équipe des sujets d'un temps $\mathrm{T} 1$ à un temps $\mathrm{T} 2$. Ces résultats confirment ainsi l'hypothèse générale formulée. Par cette confirmation notre étude valide dans le sens de F.H. Allport(1924) qui soutien que la coopération est un outil de « facilitation sociale pour désigner l'effet positif du groupe, sur les acquisitions des personnes le constituant». Pour cet auteur, «cet effet facilitateur dans ces situations de coaction est dû à deux facteurs essentiels : 
1/ La vue des mouvements effectués par autrui accroîtrait la production des mouvements du sujet (par imitation), stimulerait également la créativité et l'imagination.

2/ La présence d'autrui serait source d'une rivalité, d'une compétition entre les sujets, ce qui aurait une incidence directe sur la motivation et améliorerait les performances. Cette rivalité se traduit par l'envie de faire mieux mais aussi et souvent par l'envie de ne pas faire moins bien ». Partant de ce qui précède, l'on est en droit d'affirmer que :

« contrairement à la compétition, le coopération est fondamentalement positive. Dans les situations de nature coopérative, les comportements de domination sont atténués par le besoin d'écouter les idées d'autrui, le sentiment qu'on est responsable les uns des autres et permettent de contrer les tendances à l'individualisme et à la compétition, toujours très présentes dans le système scolaire. La pédagogie de la coopération facilite l'entraide, la confiance mutuelle, la bienveillance, l'argumentation...» (Ecole Alfitra, 2015).

Cette vision est partagée par Agathe, 2016), (Auregan, 2018) et nousmême (Koudou et Sadia, 2016).

En ce qui concerne l'ancrage interactionniste, elle est mise en évidence par Deschryver, (2008) qui présente l'autre comme «facilitateur des apprentissages et levier d'entraide entre pairs, accessibilité cognitive et rapidité de feedback ». Une telle appréciation des choses corrobore l'œuvre de Vygotski pour qui, « l'apprentissage donne donc naissance, réveille et anime chez l'enfant toute une série de développements internes qui, à un moment donné, ne lui sont accessibles que dans le cadre de la communication avec l'adulte et la collaboration avec les camarades, mais qui une fois intériorisés, deviendront une conquête propre de l'enfant... le trait fondamental de l'apprentissage consiste en la formation d'une zone proximale de développement $\gg$ (Vygotsky 1985).

En définitive, il convient de retenir que les activités de coopération sont indispensables dans le processus de rééducation des enfants en conflit avec la loi. Elles permettent d'améliorer la qualité des relations sociales, l'adoption de comportements responsables et enfin, la capacité à travailler en équipe chez des mineurs privés de liberté. Ces résultats suggèrent qu'il est temps de sortir de la logique de la «morale d'autorité et de contrainte». Cette morale marquée par une inculcation autoritaire de règles de discipline, exclut, selon Piaget, toute chance de « coopération et de réciprocité » qui dans le domaine de l'enfance délinquante semble indispensable pour induire le changement de comportement. Une telle approche débouche le plus souvent sur le mimétisme et le conformisme, sans un véritable changement de comportement. Bien au contraire, il faut œuvrer au développement de l'autodiscipline en s'accordant avec Kohlberg cité par Galichet, $(2004$, p.) pour dire qu' «aucun prêche s'il 
ne favorise pas des échanges et des discussions entre égaux, ne peut transformer la mentalité (morale) de l'adolescent ». En un mot, il faut « passer d'une approche éducative centrée sur l'information, à une approche éducative centrée sur les attitudes et les comportements dans un climat libéral de participation » (Tourette-Turgis, 2003).

\section{References:}

1. Abric, J.-C. (2019). Psychologie de la communication. Théories et méthodes. Dunod, «Psycho Sup », 192 pages.

2. Agathe, F. (2016). Les déterminants des compétences sociales des élèves de cycle III.

3. Recherches en éducation, Université de Nantes, pp.150-171. ffhalshs$01374595 f$

4. Agathe, F. (2017). " L'effet de l'accompagnement scolaire sur le développement des compétences sociales des élèves de cycle III », Recherches en éducation [En ligne], $30 \mid 2017$, mis en ligne le 01 novembre 2017, consulté le 07 novembre 2020. URL : http://journals.openedition.org/ree/2862 ; DOI https://doi.org/10.4000/ree.2862

5. Allport, F. H. (1920). The influence of the group upon association and thought; Journal of experimental psychology. 3, 159-182.

6. Allport, F. H. (1924). Social Psychology. Boston: Houghton-Mifflin,

7. Auregan, L. (2018). Devenir élève et vivre ensemble en EPS Influence de la structuration de l'apprentissage coopératif sur les relations filles garçons en danse, Mémoire Master «Métiers de l'enseignement, de l'éducation et de la formation " Mention 1, UNIVERSITE DE ROUEN ESPE - ACADEMIE DE ROUEN.

8. Baudrit, A. (2007). Apprentissage coopératif/apprentissage collaborative: d'un comparatisme conventionnel à un compparatisme critique, «Les Sciences de l'éducation - Pour l'Ère nouvelle » 1 Vol. 40, pages 115 à 136.

9. Casey, A., Dyson B. (2012). Cooperative Learning in Physical Education: A Research-Based Approach, Routledge, ISBN- 978-0415-66738-8.

10. Dennery, M. (2014). Concept \& principes pédagogiques : le conflit socio cognitif, https//www.blog-formation-entreprise.fr/conceptpedagogique-principesdaction-2-le-conflit-socio-cognitif/

11. Deutsch, M. (1949). A experimental study of the effects of cooperation and competition upon group process. Human Relations, 2, pp. 199-237. 
12. Deutsch, M. (1962). Cooperation and trust: Some theorical notes. In JONES M.R. (ed.). Nebraska symposium on motivation. University of Nebraska Press, pp. 275-319.

13. Deschryver, N.(2008). Interaction sociale et expérience d'apprentissage en formation hybride. Education. Université de Genève; University of Geneva, Français. fftel-00338100

14. Deway, J. (1927). The school and society. Chicago, The University of Chicago Press.

15. Ecole, A. (2015). Pourquoi faisons nous travailler nos élèves en binôme ou en groupe ?, www.alfirtra.over-blog.com, consulté le 3 Mars 2021.

16. Epinoux, N. et Lafont F. L (2014). Développer les compétences sociales par l'apprentissage coopératif au collège : apprendre à collaborer pour réaliser un projet collectif en EPS et en sciences physiques, Formation et profession 22(3), doi:10.18162/fp.2014.197.

17. Galichet, F. (2004). Philosophie de l'éducation, Note de Cours, Université de Franche Comte.

18. Koudou, O. et Sadia, M. A. (2016). Délinquance juvenile en Côte d'Ivoire : développement, traitement institutionnel et prévention de la recidive. Journal Africain de Communication Scientifique et Technologique, Abidjan : GRPC- IPNETP, pp.5175-5187.

19. Lewin, K. (1944). Dynamics of group action. Educational Leadership, 1, pp. 195-200.

20. Panitz, T. (1999). Cooperative versus collaborative Learning: A comparison of two concepts which will help us understand underlying nature of interactive Learning. U S Départent of Education (ERIC \# ED 448443).

21. Sadia, M. A. (2019). Etats des lieux de la pratique de la supervision pédagogique dans les Centres Privatifs de Liberté pour enfants en Côte d'Ivoire, Annales de l'Université de Moundou, Série A- Faculté des Lettres Arts et Sciences Humaines, Vol. 6(1), Oct. 2019, ISSN 23041056.

22. Sophie, M. (2015). Les compétences sociales : quels apports dans la compréhension des différences de réussite à l'école primaire ? ffhalshs-01111163f

23. Sylvain, C. (2016). «Autonomie, responsabilité et coopération: ce qu'en disent les élèves utilisant un plan de travail », Éducation et socialisation [En ligne], 41|2016, mis en ligne le 20 juillet 2016, consulté le 20 juillet 2021. DOI :

https://doi.org/10.4000/edso.1725Tourette- 
24. Tourette-Turgis, C. (1996). Le counseling, Paris: PUF, Coll. Que Saisje?

25. VYGOTSKY, L. S.(1985). « Le problème de l'enseignement et du développement mental à l'âge scolaire »-in Schneuwly \&. Bronckart (éds.). Vygotsky aujourd'hui (95-117). 